\title{
Peanuts and Their Nutritional Aspects-A Review
}

\author{
V. S. Settaluri ${ }^{1,2}$, C. V. K. Kandala ${ }^{3}$, N. Puppala ${ }^{1}$, J. Sundaram ${ }^{4}$ \\ ${ }^{1}$ New Mexico State University, Las Cruces, USA; ${ }^{2}$ Department of Biotechnology, K. L. University, Guntur, India; ${ }^{3}$ National Peanut \\ Research Laboratory, United States Department of Agriculture, Agricultural Research Service, Dawson, USA; ${ }^{4}$ United States De- \\ partment of Agriculture, Agricultural Research Service, Athens, USA. \\ Email: chari.kandala@ars.usda.gov
}

Received September $1^{\text {st }}$, 2012; revised November $16^{\text {th }}$, 2012; accepted November $23^{\text {rd }}, 2012$

\begin{abstract}
Peanut is a legume crop that belongs to the family of Fabaceae, genus Arachis, and botanically named as Arachis hypogaea. Peanuts are consumed in many forms such as boiled peanuts, peanut oil, peanut butter, roasted peanuts, and added peanut meal in snack food, energy bars and candies. Peanuts are considered as a vital source of nutrients. Nutrition plays an important role in growth and energy gain of living organisms. Peanuts are rich in calories and contain many nutrients, minerals, antioxidants, and vitamins that are essential for optimum health. All these biomolecules are essential for pumping vital nutrients into the human body for sustaining normal health. This paper presents an overview of the peanut composition in terms of the constituent biomolecules, and their biological functions. This paper also discusses about the relationship between consumption of peanuts and their effect on human metabolism and physiology. It highlights the usefulness of considering peanuts as an essential component in human diet considering its nutritional values.
\end{abstract}

Keywords: Peanuts; Nutrition; Biomolecules; Vitamins; Minerals; Plant Protein

\section{Introduction}

Peanuts are believed to have originated in Central American region from where they spread to other parts of the world. They are widely cultivated in India, Africa, South America, United States [1], China [2] and a few other countries. Peanuts often are enriched with health benefiting nutrients that are beneficial to human health. They are actually legumes but are the most frequently eaten "nut" in the United States. Studies show that peanuts, peanut butter, and peanut oil significantly reduce the risk of heart disease when consumed daily, similar to other nuts. More than 300 different varieties of peanuts are grown worldwide, which include Virginia, Valencia, Georgia runner, Tennessee red, Tennessee white and many others. They are usually consumed after roasting or boiling, and also processed into different forms such as peanut butter, candy, chocolates, cakes, and others. Peanut butter and jelly sandwiches are popular in the American culture [3], with raw, roasted, shelled or unshelled forms of peanuts being available in United States throughout the year. Peanuts vary in color from red to brown and are usually coarse in their appearance. Raw peanuts and peanuts prepared without salt are naturally low in sodium, having $18 \mathrm{mg}$ of sodium per $100 \mathrm{~g}$. This equates to only $5.4 \mathrm{mg}$ of salt in a $30 \mathrm{~g}$ serving. The nutritional importance of peanuts is due to the energy and growth supplementing constituents present in them. These include carbohydrates, lipids, proteins, vitamins, minerals, some organic acids and purines. It is estimated that as much as $30 \%$ of the population from many countries in the world are suffering from malnutrition [4]. Peanuts, which are a rich source of protein and essential amino acids, can help in preventing malnutrition [5]. Moreover, peanuts contain lipids and carbohydrates which are energy rich compounds, capable of complementing the basic energy demands of the human body. In this article the chemical composition of peanuts, all types, dry-roasted, without salt is reviewed, and their importance as a useful source of nutrition is discussed.

\section{Broad Composition of Dry-Roasted Peanuts, without Salt}

The basic composition of peanuts per $100 \mathrm{~g}$ of nuts [6] is as below:

- Water $1.55 \mathrm{~g}$

- Carbohydrates $21.51 \mathrm{~g}$

- Fiber $8.0 \mathrm{~g}$

- Lipids (Fats) $49.66 \mathrm{~g}$

- Proteins $23.68 \mathrm{~g}$ Energy (Total Calories) 2448 kJ (585 kcal)

Table 1 given below describes the sub composition of the above categories under every major molecule such as 
Table 1. Composition by weight ${ }^{*}$ of biomolecules in peanuts [6].

\begin{tabular}{|c|c|c|c|}
\hline Amino acids & & Vitamins & \\
\hline Type & Weight (g)* & Type & Weight (g) ${ }^{*}$ \\
\hline Tryptophan & 0.230 & Thiamin & $0.438 \times 10^{-3}$ \\
\hline Threonine & 0.811 & Riboflavin & $0.098 \times 10^{-3}$ \\
\hline Isoleucine & 0.833 & Niacin & $13.525 \times 10^{-3}$ \\
\hline Leucine & 1.535 & Pantothenic acid & $1.395 \times 10^{-3}$ \\
\hline Lysine & 0.850 & B6 & $0.256 \times 10^{-3}$ \\
\hline Methioione & 0.291 & Folate & $1450 \times 10^{-6}$ \\
\hline Cysteine & 0.304 & $\mathrm{E}^{* *}$ & $6.93 \times 10^{-3}$ \\
\hline Phenylalanine & 1.227 & Choline & $55.3 \times 10^{-3}$ \\
\hline Tyrosine & 0.963 & Minerals & \\
\hline Valine & 0.993 & Calcium & $54 \times 10^{-3}$ \\
\hline Arginine & 2.832 & Iron & $2.26 \times 10^{-3}$ \\
\hline Histidine & 0.599 & Magnesium & $176 \times 10^{-3}$ \\
\hline Alanine & 0.941 & Phosphorous & $358 \times 10^{-3}$ \\
\hline Aspartic acid & 2.888 & Potassium & $658 \times 10^{-3}$ \\
\hline Glutamic acid & 4.949 & Sodium & $6 \times 10^{-3}$ \\
\hline Glycine & 1.427 & Zinc & $3.31 \times 10^{-3}$ \\
\hline Proline & 1.045 & Copper & $0.671 \times 10^{-3}$ \\
\hline Serine & 1.167 & Manganese & $2.083 \times 10^{-3}$ \\
\hline Lipids & & Selenium & $7.5 \times 10^{-6}$ \\
\hline Fatty acids (total saturated) & 6.893 & Others & \\
\hline Fatty acids (total monounsaturated) & 24.640 & Carbohydrates (total) & 21.51 \\
\hline \multirow{2}{*}{ Fatty acids (total polyunsaturated) } & \multirow{2}{*}{15.694} & Dietary fiber & 8.0 \\
\hline & & Sugars (total) & 4.18 \\
\hline
\end{tabular}

*Quantities expressed are per $100 \mathrm{~g}$ of dry-roasted peanuts, all types, and without salt, throughout the paper; ${ }^{* *}$ This is a fat soluble vitamin and the rest are water soluble vitamins.

different minerals, vitamins [6], amino acids present in proteins, lipids comprising of various saturated and unsaturated fatty acids, carbohydrates comprising of polysaccharides in the form of starch, organic acids, and purines, present in peanuts.

\section{Peanut Nutritional Contribution in Comparison to Other Nuts}

Figures 1(a) and (b) shown below, explain the nutritional contribution of peanuts in comparison to other nuts and are found to add significantly more nutrients to the diet each day when compared with other nuts. This data, on the average intake of vitamins and minerals for all nut users calculated from 2001-2004 and compiled by the National Health and Nutrition Examination Survey (NHANES) was presented at the 2007 Nuts \& Health Symposium at the USDA Western Human Nutrition Research Center at the University of California, Davis. Based on a 2000 calorie intake the nutrient contribution of each nut is shown in the figures for men and women above the age of 19 years. (Courtesy: WWEIA, NHA-NES 2001-2004).

\section{Proteins in Peanuts}

It is common knowledge that all meat, chicken, turkey, fish and dairy foods are rich in proteins. Foods from animal sources provide all the protein needed by human body, which means they contain all the essential amino acids. Apart from meat, legumes such as beans, peas, and peanuts contain notable quantities of protein, more than any other nut. All amino acids [7] must be present evenly in the body to supplement normal and active growth. Among the 20 amino acids 11 are non essential, and are continuously synthesized in the body through various reactions interlinking carbohydrate and lipid metabolism. The other 9 amino acids happen to be essential ones which, ironically, cannot be synthesized by the body by itself, and hence are needed to be supplied through diet. So far it is known that, those proteins that contain all the essential amino acids in the required proportions are the complete proteins, such as casein (milk protein) and al- 


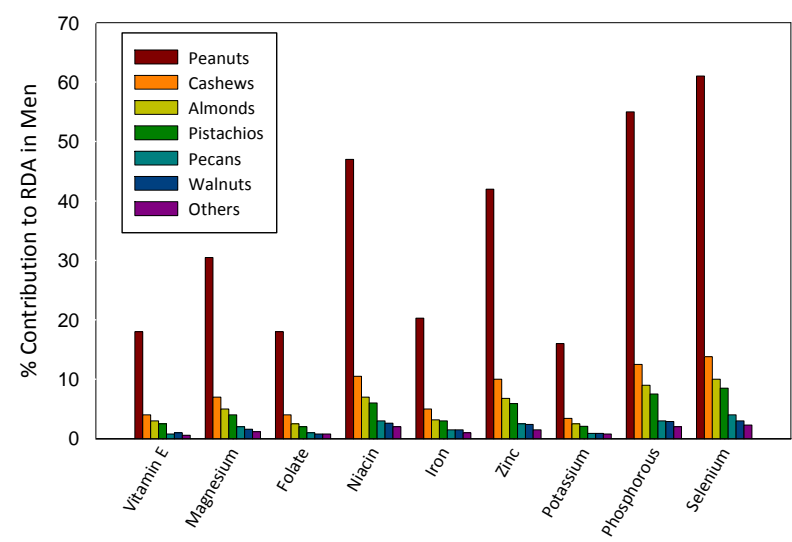

(a)

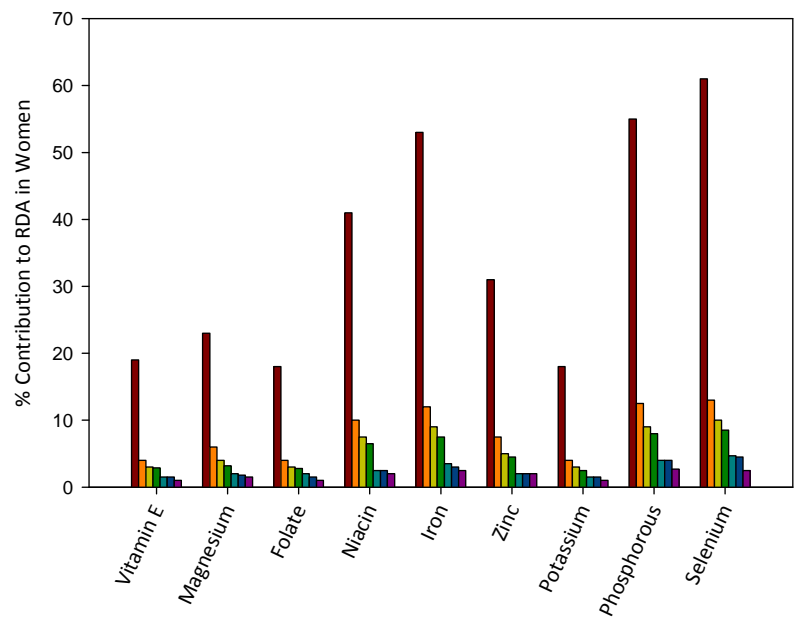

(b)

Figure 1. Peanut, peanut butter and nut nutrient contribution to recommended dietary allowances (RDA) in men (a) and in women (b) per 2000 Calories. Source and courtesy: WWEIA, NHANES 2001-2004, 1 day, 19+ years.

bumin (egg white protein). In addition to milk and eggs another vital source of protein is peanuts. Peanuts contain all the essential amino acids necessary for normal body growth and metabolism [8]. For example, lack of tryptophan in the diet may lead to pellagra, which is a very severe skin disorder, sometimes fatal [9]. Similarly, phenylalanine another essential amino acid is associated with formation of epinephrine and norepinephrine, which are also called flight or fight hormones produced by the adrenal medulla and the brain [10]. Methionine is another essential amino acid for the synthesis of protein, and it acts as an initiating amino acid during translation of proteins. Tyrosine, another amino acid is associated with formation of melanin and thyroid hormones. All these amino acids are essential to prevent protein calorie malnutrition disorders such as Kwashiorkor [11] and Marasmus [12]. Peanuts contain the above mentioned essential amino acids that are necessary for synthesis of proteins, and thus could be an indispensable component of human diet [13].
In many African countries often deaths are reported as due to malnutrition, and they could possibly be prevented by providing a protein rich diet [14]. Peanut and peanut added foods could provide such a nutritious diet. The world health organization recommends [15] an "average requirement" of $0.66 \mathrm{~g}$ of protein per $\mathrm{kg}$ of ideal body weight, and a "safe level" of $0.86 \mathrm{~g} / \mathrm{kg}$ of body weight. According to a study peanuts contain more plant protein than any other legumes or nuts [16]. Peanut are sometimes called a poor man's protein as they are available at an affordable price.

\section{Carbohydrates in Peanuts}

Roasted peanuts contain 21.51 g of carbohydrates per $100 \mathrm{~g}$ [6]. The major carbohydrate present in peanuts is starch which is a homopolysaccharide made up of $\alpha$-D glucose residues joined together by glycosidic bonds. When starch undergoes enzymatic degradation in the body by the action of amylase (present in human saliva), it is initially broken down to maltose and isomaltose [17]. These two disaccharides, when subjected to respective digestive enzymes, are converted to glucose and liberate energy. In countries such as India, the staple foods are mostly starch containing foods such as rice and potatoes. Consuming large quantities of these foods could mitigate hunger but may not provide the vital nutrients required for proper sustenance. Addition of a small quantity of peanuts to the regular diet can enhance nutrition value of a meal. The importance of the carbohydrate-amino acid interaction in the development of roasted peanut flavor and color is well documented. Carbohydrates are biomolecules, which are differentiated based on their properties as sugars and non-sugars, and the sugars are poly hydroxyl aldehyde/ketones, soluble in water, crystalline in appearance, and can be easily digested. The most abundant carbohydrate present in the human body is glucose. All the reactions associated with the metabolism of carbohydrates either begins with glucose or ends up in glucose. Glucose in the form of glycogen is stored in the liver and muscle. In the muscle, carbohydrates are utilized for doing work and in the liver they are stored as glycogen and released as and when necessary, when the body demands additional glucose, to regulate vital metabolic processes. Peanuts have adequate amounts of carbohydrates amounting to $15 \%$ of the RDA value. Carbohydrates play a critical role in supplementing valuable nutrition to the human body.

\section{Lipids in Peanuts}

Fatty acids that are present in peanuts belong to the category of lipids. In addition to carbohydrates, they also are considered as energy producing biomolecules. However, owing to their insoluble nature in water, they are not di- 
rect source of energy to the body, but act as an alternate fuel to the body. Just like carbohydrates, proteins and amino acids, lipids must be supplemented in the diet for vital biological functions. Saturated fatty acid is necessary for storage of fat soluble vitamins like vitamin A [18]. When $1 \mathrm{~g}$ of palmitic acid undergoes complete degradation by beta oxidation pathway, it can produce 9 kilo calories of energy. Fatty acids are long chain or short chain organic acids with a terminal carboxylic acid group. Certain fatty acids like palmitic and oleic can be synthesized in the body, while fatty acids like the poly unsaturated fatty acids (PUFA) need to be given through the diet. The human body does not have any mechanism for synthesizing poly unsaturated fatty acids $[19,20]$, the essential fatty acids that include, linoleic acid (18 Carbon atoms with 2 double bonds, $\mathrm{C}_{17} \mathrm{H}_{31} \mathrm{COOH}$ ), and linolenic acid (18 Carbon atoms with 3 double bonds, $\mathrm{C}_{17} \mathrm{H}_{29} \mathrm{CO}-$ $\mathrm{OH})$. These fatty acids must therefore have to be supplied from external sources through the diet. In terms of fatty acids present, edible part of roasted peanuts have $6.893 \mathrm{~g}$ saturated fatty acids, $24.64 \mathrm{~g}$ monounsaturated fatty acids (oleic acid) and $15.694 \mathrm{~g}$ polyunsaturated fatty acids. The presence of high mono and poly unsaturated fatty acids reduces the risk of coronary heart diseases. Hence peanuts and peanut oil can serve as a healthy source of nutrition [21].

\section{Vitamins in Peanuts}

Vitamins are a group of organic compounds that are not synthesized in the body, but have to be supplied to the body to maintain normal health and metabolism. They are classified as fat soluble and water soluble vitamins. Fat soluble vitamins are A, D, E, and K, and water soluble vitamins are B-Complex and vitamin C. Fat soluble vitamins play an important role in maintaining certain essential physiological functions. For example, vitamin A is associated with normal vision and epithelial functions, vitamin $\mathrm{D}$ is associated with calcium metabolism, and vitamin $\mathrm{K}$ for coagulation of blood. Water soluble vitamins are associated with certain biochemical reactions by forming coenzymes that assist the enzyme in converting a substrate into a product. For instance Riboflavin (vitamin $B_{2}$ ) in the form of Flavin mono nucleotide (FMN) and Flavin adenine dinucleotide (FAD), participate in electron transfer and hydrogen transfer reactions in the human body. All anabolic (reactions involving synthesis of carbohydrates, lipids and proteins), and catabolic (reactions involving breakdown of carbohydrates, lipids and proteins) reactions in the human body require coenzyme derivatives of B complex vitamins which are water soluble. Peanuts are a vital source for introducing most of the water soluble vitamins into the human body along with vitamin E which is fat soluble (Table 1).
An important fat soluble vitamin in peanuts is vitamin E [22] amounting to about 6.93 mg $^{*}$ (Table 1). Vitamin $\mathrm{E}$ is known as alpha-tocopherol, and is an anti-oxidative vitamin [23]. It is associated with obstructing the formation of free radicals by preventing the oxidation of unsaturated fatty acids present in consumed foods, respiratory membranes, and mitochondrial matrix in the human body. Vitamin E is associated with oxidative phosphorylation reactions in the mitochondria leading to formation of adenosine tri phosphate, known as ATP (energy currency of the cell associated with driving the metabolism of carbohydrates, proteins, and lipids). It also aids in preventing the oxidation of hemoglobin and red blood corpuscles that help intracellular respiration and adequate exchange of gases. Peanuts, in general, and peanut oil in particular, are efficient sources for supplementing vitamin $\mathrm{E}$ to the body.

Peanuts are a good source of Thiamine $\left(B_{1}\right)$, a water soluble vitamin [24], having thiamine pyrophosphate as the coenzyme, and playing a very important role in oxidative decarboxylation and co-carboxylation reactions associated with carbohydrate and amino acid metabolism. It is known that deficiency of thiamine leads to beri-beri, a disease that can cause problems in the cardiovascular and central nervous system (CNS). Peanuts contain 0.438 $\mathrm{mg}^{*}$ of thiamine (Table 1).

Peanuts are also an efficient source of Vitamin $B_{2}$. Sometimes referred to as Warburg's yellow enzyme, it has two derivatives namely FMN and FAD [25] that act as coenzymes playing an important role in electron transfer reactions, and as carriers of electrons in the electron transport chain (chain of reactions converting oxygen to water through a series of electron transfer agents). They also act as coenzymes for enzymes called oxidases, and thus participate in carbohydrate, lipid and protein metabolism. It is now known that a deficiency of vitamin $\mathrm{B}_{2}$ leads to Cheliosis (fissures at angles of the mouth), angular stomatosis (throat infection) and mouth ulcers $[26,27]$. These could be prevented by taking a diet [28] rich in vitamin $B_{2}$, and peanuts are a potential source of vitamin $\mathrm{B}_{2}$, containing $0.098 \mathrm{mg}^{*}$ of riboflavin.

Another important vitamin which is supplemented in the body by the intake of peanuts is vitamin $B_{3}$ [29] (known as Niacin or its corresponding amines, Niacinamide or Nicotinamide), to an extent of $13.525 \mathrm{mg}^{*}$. This vitamin plays an important role in the normal functioning of the respiratory chain and participates in hydrogen transfer, and electron transfer reactions through its coenzymes, Nicotinamide adenine dinucleotide (NAD) and Nicotinamide adenine dinucleotide phosphate (NADP). Deficiency of niacin leads to Pellagra, a disease characterized by diarrhea, dementia and dermatitis [30]. Tryptophan is an essential amino acid associated with the formation of niacin and has to be supplied from dietary 
sources, and incidentally peanuts are a good source of tryptophan $\left(0.230 \mathrm{~g}^{*}\right)$.

Vitamin $B_{5}$, found in many food sources, needs a strong presence in human diet owing to its widespread applications in numerous biochemical reactions within the human body. $\mathrm{B}_{5}$ is found in good amounts in peanuts. Peanuts, mushrooms, soybeans, split peas, pecans, oatmeal, buckwheat, sunflower seeds, lentils, rye flour, cashews, and other whole grains and nuts are good sources of pantothenic acid. Chemically Vitamin $\mathrm{B}_{5}$ is a dipeptide consisting of pantoic acid and beta alanine, and as a whole known as pantothenic acid (pantothenate in Greek means from everywhere), which is an important constituent of peanuts, present to the extent of $1.395 \mathrm{mg}^{*}$ (Table 1). Pantothenic acid in humans is responsible for the formation of Coenzyme A, which is responsible for many vital reactions in the body such as the kreb's cycle, synthesis of cholesterol [31], and synthesis of heme.

Another important water soluble vitamin, a notable constituent of Peanuts, is Vitamin $\mathrm{B}_{6}$, Pyridoxine. Vitamin $B_{6}$ occurs in three naturally occurring forms namely pyridoxine, pyridoxalamine, and pyridoxal. It has two coenzyme forms, pyridoxal phosphate and pyridoxal amine phosphate, and these two forms carry out many significant biochemical reactions in the body especially those associated with amino acid metabolism. It is highly essential to supplement this vitamin through the diet into the human body, and roasted peanuts will provide $\mathrm{B}_{6}$ to the human body to the extent of $0.256 \mathrm{mg}^{*}$.

Vitamin B9, more commonly known as folate or folic acid, is a water-soluble vitamin that is part of the B vitamin family. Folate occurs naturally in foods, while folic acid is the synthetic form of folate. Vitamin B9 is useful for human growth and helps the mechanism of normal nerve and brain functioning [32]. B9 is also credited with controlling blood-levels of the amino acid homocysteine, thus reducing the risk of heart disease and stroke. Folate present in peanuts to an extent of $145 \mathrm{\mu g}^{*}$ may also help protect against cancers of the lung, colon, and cervix.

\section{Minerals in Peanuts}

Magnesium is an essential mineral for the human body for several useful functions [33]. Magnesium is needed to maintain normal muscle and nerve functions and a healthy immune system. Magnesium promotes normal blood pressure, keeps bones strong, and helps to regulate blood sugar levels. People who consume peanuts have shown higher levels of magnesium. Peanuts are a good source of magnesium containing $176 \mathrm{mg}^{*}$ (Table 1).

A mineral that is primarily associated with strengthening of bones, gums and teeth is calcium. Calcium plays an important role in the normal functioning of the visual cycle, and in the mechanism of blood coagulation. It is also associated with normal muscle physiology, helping in contraction and relaxation of muscles. Though, mainly present in milk, it is also present to a notable extent in peanuts containing $54 \mathrm{mg}^{*}$ (Table 1 ).

A mineral that aids primarily in the formation of bones and teeth along with calcium is phosphorous. Phosphorous helps to synthesize protein for the growth, maintenance and repair of cells, and tissues. With $358 \mathrm{mg}^{*}$ (Table 1) of phosphorous, peanuts are a good source of phosphorous providing about $50 \%$ of our daily needs.

One has to eat the right amount of foods that contain potassium to keep the blood levels in a healthy range. Maintaining fluid and electrolyte balance is a critical function in the human body and this function is taken care of by potassium. In association with sodium ions, potassium plays an important role in the brain and nerve functions, and for muscle development. Peanuts containing $658 \mathrm{mg}^{*}$ of potassium are a good source of this vital mineral (Table 1).

Zinc is a cofactor for most of the dehydrogenases that require the coenzymes NAD and NADP. This mineral also supports our immune systems, helps in wound healing, and is involved in building proteins. Zinc is an essential mineral for normal growth and development during pregnancy, childhood, and adolescence. Zinc also helps the cells in human body communicate by functioning as a neurotransmitter. Deficiency of zinc can lead to stunted growth, diarrhea, impotence, hair loss, eye and skin lesions, impaired appetite, and depressed immunity. Peanuts are a great source of zinc. One hundred grams of roasted peanuts will provide $3.31 \mathrm{mg}^{*}$ of zinc (Table 1).

Peanuts contain $2.26 \mathrm{mg}^{*}$ of iron (Table 1). Iron is an essential metal ion that acts as the chelating agent, and chelates with heme to form hemoglobin. It is an integral part of many enzymes that participate in biochemical reactions such as peroxidase, catalase and ferro chelatase. It is involved in oxygen transport and helps regulate cell growth and differentiation. It is known that the deficiency of iron leads to anemia which is prevalent in many parts of the world [34].

Copper plays a role in the production of key proteins in our body such as collagen and hemoglobin, which trans- port oxygen. A serving of one hundred grams of roasted peanuts provide $0.671 \mathrm{mg}^{*}$ (Table 1), and thus peanuts are an excellent source of copper providing over $70 \%$ of our daily needs [35].

Selenium is an essential mineral but needed in small amounts for the human body. Selenium is naturally found in plants, seafood, meat and meat products. Selenium is a micronutrient and antioxidant, and a component of several essential enzymes. Selenium is believed to delay or prevent the onset of cancer and also has anti-aging effects [36]. Selenium is found in peanuts to the extent of $7.5 \mathrm{\mu g}^{*}$ (Table 1) giving about $14 \%$ of our daily needs. 


\section{Raw and Roasted Peanuts}

Though unroasted peanuts are fit for consumption, they do not have the same flavor as the roasted ones. However, a significant risk in eating raw peanuts is the possible presence of aflatoxin [37], a poison produced by a fungus, though it may occur rarely. Roasting peanuts usually removes this fungus. Eating contaminated peanuts can cause liver diseases.

It was found in $0.4 \%$ to $0.6 \%$ of persons, particularly in children, consumption of peanuts in any form lead to allergic reactions, some severe. These may develop in the childhood or could be due to a family history of peanut allergy [38]. The symptoms associated with peanut allergy may range from oozing and skin rashes, to shortness of breath or wheezing.

Consumption of nuts has a positive effect on coronary heart diseases. It has been observed that the risk of coronary heart diseases and related cardiovascular diseases [39] is $37 \%$ lower for those consuming nuts more than 4 times per week, than in those people who don't consume nuts at all.

Peanuts play a crucial role in weight management [40]. Epidemiological studies have provided useful information on beneficial effects of nuts which also includes peanuts. Even though peanuts are rich in fats, they can still be included in diet for weight loss.

\section{Conclusion}

Peanuts are an excellent and affordable source of nutriation, supplementing vital nutrients to the human body such as proteins, carbohydrates, lipids, vitamins, minerals and fiber. Peanuts are often referred as poor man's protein but, when taken in adequate amounts in any form, will supplement rich nutrients to the body that can provide growth and energy, and play a vital role in the prevention of diseases. Metal ions which are cofactors for many enzymes, vitamins that are not synthesized in the body, poly unsaturated and monounsaturated fatty acids, whose consumption can increase the levels of HDL cholesterol which is good for the heart, are present in peanuts. Essential amino acids that are not synthesized in the body, but are essential as building blocks of proteins and carbohydrates, which are the principle source of energy in the body, can be provided by peanuts. A diet including peanuts could provide all these vital nutrients, and play a critical role in preventing disease and promoting good health.

\section{REFERENCES}

[1] D. D. Tom, "Earliest-Known Evidence of Peanut, Cotton and Squash Farming Found," 2007. http://www.eurekalert.org/pub_releases/2007-06/vu-eeo0 62507.php
[2] G. Yao, "Peanut Production and Utilization in the People’s Republic of China,” 2004.

http://www.caes.uga.edu/commodities/fieldcrops/peanuts/ pins/documents/ChinaProduction.pdf

[3] Unknown, "Peanuts at the World's Healthiest Food," 2007. http://www.whfoods.com/genpage.php?tname=foodspice \&dbid $=101$

[4] FAO, “The spectrum of Malnutrition,” 2000. http://www.fao.org/worldfoodsummit/english/fsheets/mal nutrition.pdf

[5] G. H. Pelto and M. Armar-Klemesu, "Balancing Nurturance, Cost and Time: Complementary Feeding in Accra, Ghana,” Maternal \& Child Nutrition, Vol. 7, No. 3, 2011, pp. 66-81.

[6] USDA, National Nutrient Database for Standard Reference, Release 24, 2011.

[7] G. Schaafsma, "The Protein Digestibility-Corrected Amino Acid Score,” Journal of Nutrition, Vol. 130, No. 7, 2000. pp. 1865-1867.

[8] J. R. Hoffmann and M. J. Falvo, "Protein-Which Is Best?” Journal of Sports Science and Medicine, Vol. 3, No. 3, 2004, pp. 118-130.

[9] A. J. Seal, P. I. Creeke, F. Dibari, E. Cheung, E. Kyroussis, P. Semedo and T. Briel, "Low and Deficient Niacin Status and Pellagra Are Endemic in Postwar Angola," American Journal of Clinical Nutrition, Vol. 85, No. 1, 2007, pp. 218-224.

[10] S. N. Young, "Behavioral Effects of Dietary Neurotransmitter Precursors: Basic and Clinical Aspects," Alternative Medicine Review, Vol. 20, No. 2, 1996, pp. 313-320.

[11] P. Shetty, "Malnutrition and under Nutrition," Medicine, Vol. 34, No. 12, 2006, pp. 524-529. doi:10.1053/j.mpmed.2006.09.014

[12] S. M. A. El Hassan, N. M. Abdelrazik, A. E. F. Abd ElAziz and R. R. El-Iraqi, "Assessment of the Relation between Trace Elements and Antioxidant Status in Children with Protein Energy Malnutrition," The Internet Journal of Pediatrics and Neonatology, Vol. 4, No. 1, 2004, pp. 25-36.

[13] E. J. Conkerton and R. L. Ory, "Peanut Proteins as Food Supplements: A Compositional Study of Selected Virginia and Spanish peanuts,” 1976. http://www. food.oregonstate.edu/ref/uken/uken_7.html

[14] T. Sanghvi and J. Murray, "Improving Child Health through Nutrition; The Nutrition 1 Minimum Package," 1997.

http://www.basics.org/documents/pdf/ImprovingChildHe althThroughNutrition.pdf

[15] Food and Nutrition Board, “Dietary Reference Intakes for Energy, Carbohydrates, Fiber, Fat, Protein and Amino Acids (Macronutrients)," The National Academy Press, Washington, 2002.

[16] Food composition. http://fnic.nal.usda.gov/nal_display/index.php?info_cente $\mathrm{r}=4 \&$ tax_level=2\&tax_subject=279\&topic_id=1553

[17] S. C. Zeeman, J. Kossmann and A. M. Smith, "Starch: Its Metabolism, Evolution, and Biotechnological Modifica- 
tion in Plants," Annual Review of Plant Biology, Vol. 61, No. 1, 2010, pp. 209-234. doi:10.1146/annurev-arplant-042809-112301

[18] J. B. German and C. J. Dillard, "Saturated Fats: What Dietary Intake?" American Journal of Clinical Nutrition, Vol. 80, No. 3, 2004, pp. 550-559.

[19] R. E. Worthington and D. H. Smith, "Modification of Peanut Oil Fatty Acid Composition by Foliar Applications of 2',2'-dimethylsuccinohydrazide (Kylar),” Journal of Agricultural and Food Chemistry, Vol. 22, No. 3, 1974, pp. 507-508. doi:10.1021/jf60193a056

[20] J. S. L. How and C. T. Young, "Comparison of Fatty Acid Content of Imported Peanuts," Journal of the American Oil Chemists' Society, Vol. 60, No. 5, 1983, pp. 945947. doi:10.1007/BF02660204

[21] American Peanut Council, "Nutritional Values, Energy and Fat, Fibre/Minerals/Vitamins/Phytochemicals/Protein.” http://www.peanutsusa.org.uk/Europe/index.cfm?fuseacti on=home.page\&pid=64

[22] R. Blomhoff, M. H. Carlsen, L. F. Andersen and D. R. Jacobs Jr., "Health Benefits of Nuts: Potential Role of Antioxidants,” British Journal of Nutrition, Vol. 99, No. 2, 2008, pp. 447-448.

[23] X. Gao, P. E. Wilde, A. H. Lichtenstein, O. I. Bermudez and K. L. Tucker, "The Maximal Amount of Dietary Alpha-Tocopherol Intake in US Adults (NHANES 20012002)," Journal of Nutrition, Vol. 136, No. 4, 2006, pp. 1021-1026.

[24] C. M. Alper and R. D. Mattes, "Peanut Consumption Improves Indices of Cardiovascular-Thiamine Disease Risk in Healthy Adults," Journal of the American College of Nutrition, Vol. 22, No. 2, 2003, pp. 133-141.

[25] S. O. Mansoorabadi, C. J. Thibodeaux and H. Liu, "The Diverse Roles of Flavin Coenzymes-Nature's Most Versatile Thespians," The Journal of Organic Chemistry, Vol. 72, No. 17, 2007, pp. 6329-6342. doi:10.1021/jo0703092

[26] H. Zhao, X. Yang, R. Zhou and Y. Yang, "Study on Vitamin B1, Vitamin B2 Retention Factors in Vegetables," Journal of Hygiene Research, Vol. 37, No. 1, 2008, pp. 92-96.

[27] D. B. McCormick, “Riboflavin,” In: M. E. Shils, J. A. Olson, M. Shike and A. C. Ross, Eds., Modern Nutrition in Health and Disease, 9th Edition, Lea \& Febiger, Williams \& Wilkins, Baltimore, 1999, pp. 391-400.

[28] R. S. Rivlin, “Riboflavin,” In: E. K. Ziegler and L. J. Filer Eds., Present Knowledge in Nutrition, 7th Edition, International Life Sciences Institute, Washington, 1996, pp. 167-173.

[29] B. G. Brown, X. Q. Zhao, A. Chalt, et al., "Simvastatin and Niacin, Antioxidant Vitamins, or the Combination for the Prevention of Coronary Disease," The New England Journal of Medicine, Vol. 345, No. 22, 2001, pp. 15831592. doi:10.1056/NEJMoa011090

[30] J. R. Guyton, "Niacin in Cardiovascular Prevention: Mechanisms, Efficacy, and Safety," Current Opinion in Lipidology, Vol. 18, No. 4, 2007, pp. 415-420. doi:10.1097/MOL.0b013e3282364add

[31] S. Kimura, Y. Furukawa, J. Wakasugi, Y. Ishihara and A. Nakayama, "Antagonism of L(-)pantothenic Acid on Lipid Metabolism in Animals," Journal of Nutritional Science and Vitaminology, Vol. 26, No. 2, 1980, pp. 113-117. doi:10.3177/jnsv.26.113

[32] S. M. Fishman, P. Christian and K. P. West, "The Role of Vitamins in the Prevention and Control of Anemia," Public Health Nutrition, Vol. 3, No. 2, 2000, pp. 125-150. doi:10.1017/S1368980000000173

[33] S. C. Larsson and A. Wolk, "More Magnesium-Rich Food for Less Diabetes," Journal of Internal Medicine, Vol. 262, No. 2, 2007, pp. 208-214. doi:10.1111/j.1365-2796.2007.01840.x

[34] S. Miret, R. J. Simpson and A. T. McKie, "Physiology and Molecular Biology of Dietary Iron Absorption,” Annual Review of Nutrition, Vol. 23, 2003, pp. 283-301. doi:10.1146/annurev.nutr.23.011702.073139

[35] Institute of Medicine, Food and Nutrition Board, "Dietary Reference. Intakes for Vitamin A, Vitamin K, Arsenic, Boron, Chromium, Copper, Iodine, Iron, Manganese, Molybdenum, Nickel, Silicon, Vanadium and Zinc,” 2001. www.nutrisci.wisc.edu/NS623/drivitasum.pdf

[36] C. D. Thomson, "Assessment of Requirements for Selenium and Adequacy of Selenium Status: A Review," European Journal of Clinical Nutrition, Vol. 58, No. 3, 2004, pp. 391-402. doi:10.1038/sj.ejcn.1601800

[37] E. Robert, T. Pettit and T. Ruth Ann, "Factors Influencing Aflatoxin Accumulation in Peanut Kernels and the Associated Mycoflora,” Applied Microbiology, Vol. 16, No. 8, 1968, pp. 1230-1234.

[38] M. B. Gideon Lack, F. Deborah and N. Kate, "Factors Associated with the Development of Peanut Allergy in Childhood," The New England Journal of Medicine, Vol. 348, No. 11, 2003, pp. 977-985. doi:10.1056/NEJMoa013536

[39] J. H. Kelly, Jr. and J. Sabaté, "Peanuts and Cardiovascular Disease,” British Journal of Nutrition, Vol. 96, No. 2, 2006, pp. 61-67. doi:10.1017/BJN20061865

[40] H. Jennette, "The Potential Role of Peanuts in the Prevention of Obesity," Nutrition \& Food Science, Vol. 35, No. 5, 2005, pp. 353-358. doi:10.1108/00346650510625566 